\title{
Mitral valvuloplasty complicated by catheter perforation of the right atrium and the aortic root
}

\author{
Alessandra Francica ${ }^{1}$, Francesco Onorati ${ }^{1}$, Giuseppe Faggian ${ }^{2}$, and Alfredo Vicentini ${ }^{3}$ \\ ${ }^{1}$ Integrated University Hospital of Verona \\ ${ }^{2}$ Integrated University Hospital of Verona, Division of Cardiac Surgery \\ ${ }^{3}$ Ospedale di Peschiera
}

May 18, 2020

\begin{abstract}
According with latest guidelines, percutaneous mitral commissurotomy (PMC) represents the first-line treatment for symptomatic severe mitral valve stenosis (SMVS) with favourable morphology, We report a successful surgical treatment of a potential life-threatening complication occurred during PMC. Heart-Team discussion and closed collaboration with Centres are crucial for decision-making and Cardiac Surgery onsite should be ensured for high-risk procedures.
\end{abstract}

\section{Case report}

A 77-year-old female with symptomatic SMVS was admitted to a secondary referring Hospital. Transoesophageal echocardiography demonstrated a SMVS (valve area $0,9-1 \mathrm{~cm}^{2}$ and mean gradient of $17 \mathrm{mmHg}$ ), with commissural fusion, diffused calcification of the annulus and leaflets, and no mitral regurgitation. LVEF was $60 \%$ and there were no left atrial thrombi. Severe pulmonary hypertension (PAPs $85 \mathrm{mmHg}$ ) was identified.

After Heart-Team discussion, PMC was planned according with current ESC/EACTS Guidelines ${ }^{(1)}$. The procedure was performed at the same admitting hospital with a high-volume interventional cardiology programme but no cardiac surgery onsite. After peripheral trans-venous catheterization, despite a continuous trans-oesophageal echocardiographic and radioscopic monitoring $\backslash$ sout, inadvertent catheter perforation of the right atrium and of the non-coronary sinus of the aortic root (Figure 1) was noted, resulting in transient haemodynamic decompensation.

Immediate intensive-care support allowed for haemodynamic stability, so patient could be emergently transferred to our centre and directly brought to our "stand-by" operating theatre.

Intraoperative assessment confirmed that angiographic catheter together with the supportive guide-wire perforated the roof of the right atrium, and the non-coronary sinus of the aortic root (Figure 2). Spontaneous formation of a pericardial clot prevented active bleeding. After standard aorto-bicaval cardiopulmonary bypass, aortic cross-clamping and cardioplegic arrest, the catheter and the guide-wire were removed, and the right atrial perforation was closed with a pericardial patch. After transverse aortotomy, non-coronary sinus was resected and substituted with bovine pericardial patch (XenoSure@ Biologic Patch, LeMaitre Vascular, USA), followed by a conventional $25 \mathrm{~mm}$ bioprosthetic mitral valve replacement (EPIC, St Jude Medical, Inc, St Paul, Minn). Given the extensive mitral annular calcifications, only partial posterior mitral annular decalcification was carried out, due to the risk of atrio-ventricular discontinuity and/or para-valvular leak.

Weaning from cardiopulmonary bypass support required high-dose inotropes, IABP, and inhaled NO due to persistent severe pulmonary hypertension with right ventricular dysfunction. A delayed-sternal closure 
approach was chosen to favour postoperative RV recovery.

Progressive postoperative recovery was noted, leading to sternal closure on $2^{\text {nd }}$ POD, IABP-withdrawal on $7^{\text {th }}$ POD, and extubation on $10^{\text {th }}$ POD. Transient acute kidney injury required dialysis. The patient was transferred to Rehabilitation Clinic on $48^{\text {th }}$ POD with stable hemodynamic, no inotropic support, and a recovered renal function.

The patient was discharged home on $78^{\text {th }}$ POD in healthy conditions, and she is alive at 7 months of follow-up in NYHA class I-II.

\section{Discussion}

We report here a successful surgical treatment of a potential life-threatening complication of PMC.

The treatment of SVMS has been revolutionized since the development of PMC in 1980s: trans-septal catheterization is one of the most crucial procedural steps and the Inoue balloon technique is the standard of care for PMC. ${ }^{(1)}$

According to the latest ESC/EACTS Guidelines PMC represents the first-line treatment (class I, level B) for most symptomatic SVMS and favourable morphology. ${ }^{(1)}$

However, albeit very high procedural success rates of PMC have been reported by Bauleti et al. ${ }^{(2)}$, long-term freedom from reintervention (either repeat PMC and surgery) is only about $38+-2 \%$.

In addition, $\mathrm{PMC}$ in-hospital mortality rate is up to $0.4 \%$ and the most common complications were embolic stroke and severe mitral regurgitations, occurring in $0.3 \%$ and $3.4 \%$ respectively. ${ }^{(3)}$

Finally, Iung et al. (4) reported an incidence of technical failure of $1.2 \%$, due to hemopericardium, embolism, inability to cross the septum, or inability to position the balloon across the valve.

The need for surgery was estimated to occur in $4.7 \%$ during the first post-procedural month.

As far as causes of cardiac perforation during PMC are concerned, several mechanisms have been identified ${ }^{(5)}$ 1) apical tears by straight-tip balloon catheters driven distally during mitral valve dilatation, 2) apical perforations by guidewires introduced through catheters wedged in the apex, 3) tear of the posterior right atrial wall by dilatation of the track produced by very low septal punctures, 4) right ventricular perforation by a pacing catheter, and 5) perforation of the aortic root and adjacent right atrium by sliding up of the trans-septal set. Of note, cardiac perforation was identified to be significantly related to the total experience at the Center and to patient age.

In our case, a type 5 mechanism of perforation occurred, despite the high-volume experience of the performing Centre.

In conclusion, local policies may allow risky percutaneous procedures to be performed in high-volume Cardiology Centres without cardiac Surgery onsite. Although we recommend these procedures to be "centralized" in third-level Hospitals with prompt availability of on-site Cardiac Surgery, successful surgical treatment of life-threatening complications following risky percutaneous procedures in unfavourable logistics can be achieved by a Heart Team approach providing ad-hoc availability of dedicated "stand-by" operating theatre. 


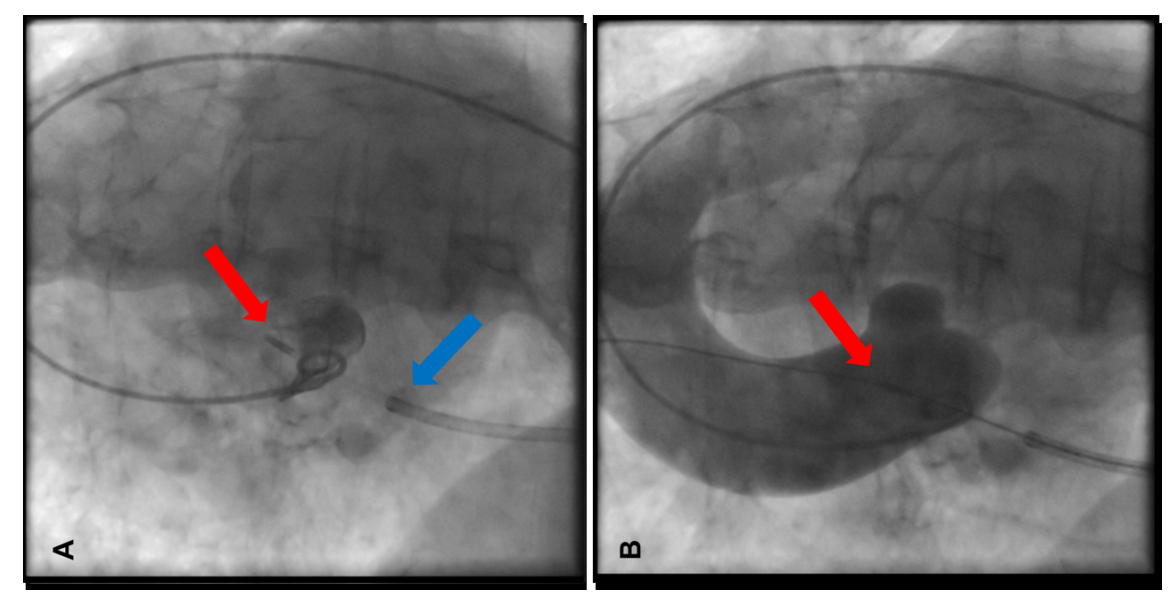



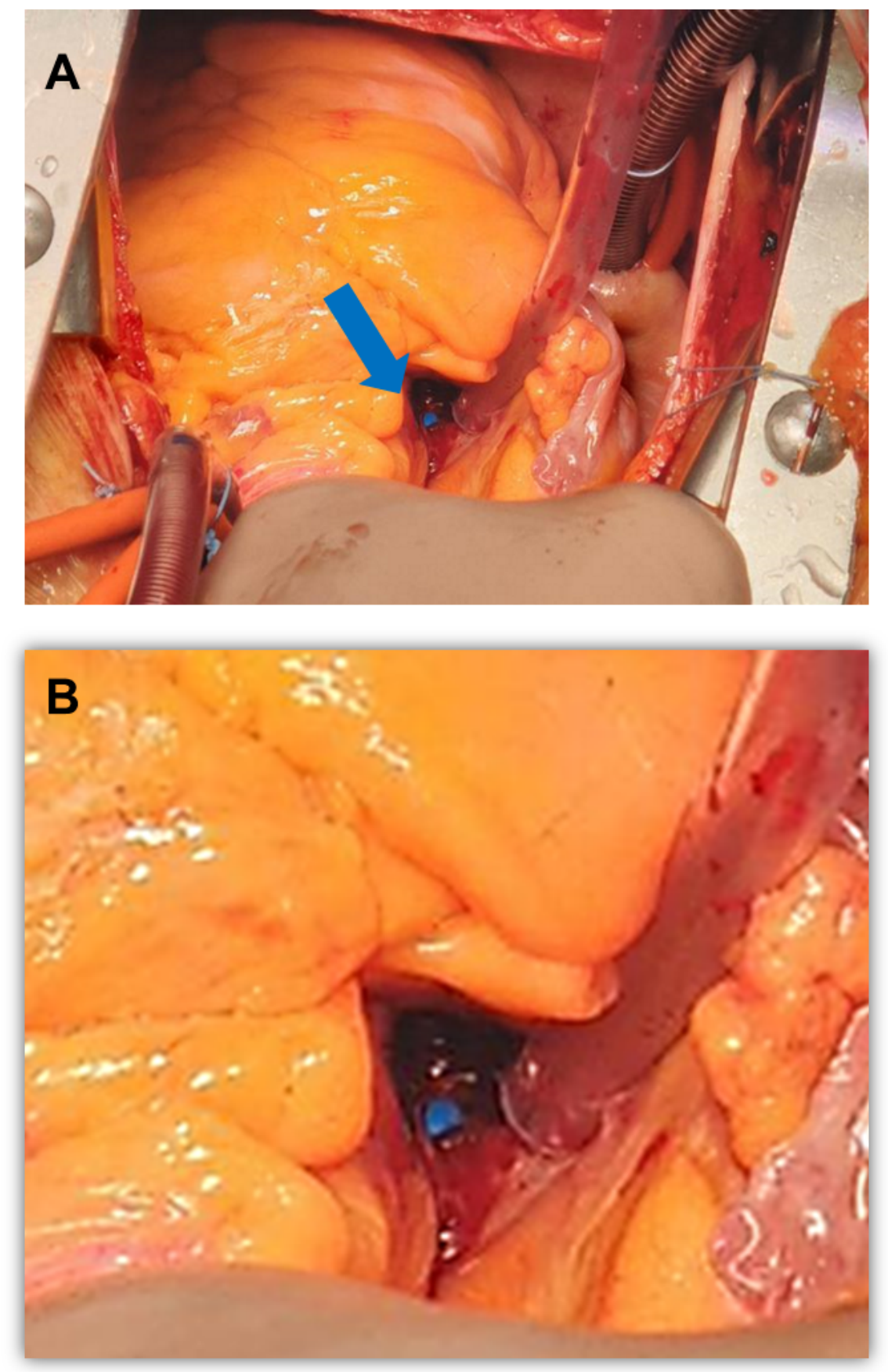

\section{Figure Legends:}

Figure 1. Angiographic view: A) Guidewire catheter (red arrow) perforating the non-coronary sinus of the aortic root and adjacent right atrium (blue arrow). B) Aortic root perforation confirmed by contrast (red arrow).

Figure 2. Surgical view: A) Guidewire catheter (blue arrow) perforation of atrium roof and aortic root. B) Detail of catheter guidewire perforation. 


\section{References}

[1] Baumgartner, H. et al. 2017 ESC/EACTS Guidelines for the management of valvular heart disease. Eur. Heart J. 38, 2739-2791 (2017).

[2] Bouleti, C. et al. Reinterventions after percutaneous mitral commissurotomy during long-term follow-up, up to 20 years: The role of repeat percutaneous mitral commissurotomy. Eur. Heart J.34, 1923-1930 (2013).

[3] Bouleti, C. et al. Late results of percutaneous mitral commissurotomy up to 20 years: Development and validation of a risk score predicting late functional results from a series of 912 patients. Circulation 125, 2119-2127 (2012).

[4] Iung, B. et al. Temporal trends in percutaneous mitral commissurotomy over a 15-year period. Eur. Heart J. 25, 701-707 (2004).

[5] Joseph, G., Chandy, S. T., Krishnaswami, S., Ravikumar, E. \& Korula, R. J. Mechanisms of cardiac perforation leading to tamponade in balloon mitral valvuloplasty. Cathet. Cardiovasc. Diagn. (1997) 